\title{
Intracranial inertial cavitation threshold and thermal ablation lesion creation using MRI-guided 220-kHz focused ultrasound surgery: preclinical investigation
}

\author{
Zhiyuan Xu, MD, ${ }^{1}$ Carissa Carlson, ${ }^{5}$ John Snell, PhD, ${ }^{5}$ Matt Eames, PhD, ${ }^{5}$ Arik Hananel, MD, MBA, ${ }^{5}$ \\ M. Beatriz Lopes, MD, PhD, ${ }^{3}$ Prashant Raghavan, MD, ${ }^{2}$ Cheng-Chia Lee, MD, ${ }^{1}$ Chun-Po Yen, MD, ${ }^{1}$ \\ David Schlesinger, PhD, ${ }^{1,4}$ Neal F. Kassell, MD, ${ }^{5}$ Jean-Francois Aubry, PhD, ${ }^{4,6}$ \\ and Jason Sheehan, MD, PhD ${ }^{1,4}$
}

Departments of ${ }^{1}$ Neurosurgery, ${ }^{2}$ Neuroradiology, ${ }^{3}$ Pathology (Neuropathology), and ${ }^{4}$ Radiation Oncology, University of Virginia Health System; ${ }^{5}$ Focused Ultrasound Foundation, Charlottesville, Virginia; and ${ }^{6}$ Institut Langevin, CNRS UMR 7587, INSERM U979, ESPCI Paris Tech, Paris, France

\begin{abstract}
OBJECT In biological tissues, it is known that the creation of gas bubbles (cavitation) during ultrasound exposure is more likely to occur at lower rather than higher frequencies. Upon collapsing, such bubbles can induce hemorrhage. Thus, acoustic inertial cavitation secondary to a $220-\mathrm{kHz}$ MRI-guided focused ultrasound (MRgFUS) surgery is a serious safety issue, and animal studies are mandatory for laying the groundwork for the use of low-frequency systems in future clinical trials. The authors investigate here the in vivo potential thresholds of MRgFUS-induced inertial cavitation and MRgFUS-induced thermal coagulation using MRI, acoustic spectroscopy, and histology.
\end{abstract}

METHODS Ten female piglets that had undergone a craniectomy were sonicated using a 220-kHz transcranial MRgFUS system over an acoustic energy range of 5600-14,000 J. For each piglet, a long-duration sonication (40-second duration) was performed on the right thalamus, and a short sonication (20-second duration) was performed on the left thalamus. An acoustic power range of 140-300 W was used for long-duration sonications and 300-700 W for shortduration sonications. Signals collected by 2 passive cavitation detectors were stored in memory during each sonication, and any subsequent cavitation activity was integrated within the bandwidth of the detectors. Real-time 2D MR thermometry was performed during the sonications. T1-weighted, T2-weighted, gradient-recalled echo, and diffusion-weighted imaging MRI was performed after treatment to assess the lesions. The piglets were killed immediately after the last series of posttreatment MR images were obtained. Their brains were harvested, and histological examinations were then performed to further evaluate the lesions.

RESULTS Two types of lesions were induced: thermal ablation lesions, as evidenced by an acute ischemic infarction on MRI and histology, and hemorrhagic lesions, associated with inertial cavitation. Passive cavitation signals exhibited 3 main patterns identified as follows: no cavitation, stable cavitation, and inertial cavitation. Low-power and longer sonications induced only thermal lesions, with a peak temperature threshold for lesioning of $53^{\circ} \mathrm{C}$. Hemorrhagic lesions occurred only with high-power and shorter sonications. The sizes of the hemorrhages measured on macroscopic histological examinations correlated with the intensity of the cavitation activity $\left(R^{2}=0.74\right)$. The acoustic cavitation activity detected by the passive cavitation detectors exhibited a threshold of $0.09 \mathrm{~V} \cdot \mathrm{Hz}$ for the occurrence of hemorrhages.

CONCLUSIONS This work demonstrates that $220-\mathrm{kHz}$ ultrasound is capable of inducing a thermal lesion in the brain of living swines without hemorrhage. Although the same acoustic energy can induce either a hemorrhage or a thermal lesion, it seems that low-power, long-duration sonication is less likely to cause hemorrhage and may be safer. Although further study is needed to decrease the likelihood of ischemic infarction associated with the 220-kHz ultrasound, the threshold established in this work may allow for the detection and prevention of deleterious cavitations.

http://thejns.org/doi/abs/10.3171/2014.9.JNS14541

KEY WORDS magnetic resonance-guided focused ultrasound; MRsFUS; inertial cavitation; swine brain; acoustic cavitation threshold; surgical technique

ABBREVIATIONS FUS = focused ultrasound; MRgFUS = MRI-guided FUS; TCMRgFUS = transcranial MRgFUS. SUBMITTED March 11, 2014. ACCEPTED September 29, 2014 INCLUDE WHEN CITING Published online November 7, 2014; DOI: 10.3171/2014.9.JNS14541.

DISCLOSURE This work was funded by a grant from the Focused Ultrasound Foundation. Drs. Hananel and Kassell have shares in InSightec, the company that manufactures the ExAblate device. Drs. Snell, Hananel, Eames, and Kassell and Ms. Carlson are paid employees of the Focused Ultrasound Foundation. Dr. Aubrey is a consultant for the Focused Ultrasound Foundation. 
$\mathrm{T}$ RANSCRANIAL MRI-guided focused ultrasound (TcMRgFUS) surgery represents a potential minimally invasive and ionization radiation-free treatment alternative to resection and radiosurgery..$^{14}$ It was made possible by implementing aberration correction techniques ${ }^{4,51}$ on multielement arrays of ultrasound transducers $^{22,35}$ to compensate for the aberrations induced by the skull bone. ${ }^{15,56}$ Successful thalamotomy of patients with medically refractory essential tremor is one promising intracranial application. ${ }^{12} \mathrm{TcMRgFUS}$ offers other potential applications, including brain tissue ablation,,$^{19,32,36,48}$ blood-brain barrier opening for therapeutic agent delivery, ${ }^{24,57}$ thrombolysis, $, 3,7$ and potentiation of the anticancer immune response. 53,58

Currently, available clinical TcMRgFUS devices operate at $650 \mathrm{kHz} .{ }^{12,36,38}$ Lower-frequency (e.g., 220-kHz ${ }^{1,11,43}$ ) devices exist but at the present time are reserved for research. There are several advantages of using a low-frequency TcMRgFUS device. First, the larger ratio of wavelength to path length in the skull bone at $220 \mathrm{kHz}$ results in reduced phase aberration, thereby eliminating the need for extensive patient-specific correction. ${ }^{60}$ Second, the small focal zone volume of the current $650-\mathrm{kHz}$ device is inefficient for the treatment of larger volumetric targets, for which many individual sonications must be delivered serially in an overlapping manner to deliver conformal thermal ablation. The low-frequency transducer offers an enlarged focal zone with a concomitant improvement in treatment efficiency. ${ }^{49}$ This increased efficiency may render feasible the treatment of larger, clinically important targets while maintaining practical treatment times.

However, low-frequency FUS increases the likelihood of acoustic cavitation in tissues. ${ }^{23}$ Cavitation results from the peak negative pressure of a propagating ultrasonic wave expanding small amounts of gas trapped in small cavities (cavitation nuclei). ${ }^{16,30}$ These small bubbles will grow from rectified diffusion and pulsate volumetrically in the presence of subsequent ultrasonic excitation. ${ }^{30}$ Two forms of acoustic cavitation exist and differ according to the type of bubble pulsatility. Stable cavitation is longlasting bubble oscillation in which the dynamics of the cavity motion are dominated by the compressibility of the gas. ${ }^{46}$ It is associated with microstreaming surrounding the bubbles that can reversibly modify the permeability of the cell membranes. ${ }^{59}$ Inertial (or transient) cavitation is a series of unstable bubble expansions followed by a rapid and violent collapse. Bubble collapse releases high levels of energy and may cause severe mechanical damage to the surrounding tissues because of its associated high internal temperature, pressure, and velocity jets. ${ }^{6,41,47}$ Bubbles oscillating under stable and inertial cavitation have distinct acoustical signatures that can be recorded by dedicated ultrasonic detectors. ${ }^{17,18,30}$

Inertial cavitation may result in thermal and/or mechanical injury to normal brain adjacent to the clinical target and may result in devastating and potentially fatal intracranial hemorrhage. The formation of gas bubbles without previous injection of microbubbles has been described ${ }^{30}$ and may have been responsible for ultrasound-related hemorrhages reported from a low-frequency $300-\mathrm{kHz}$ sonothrombolysis study. ${ }^{2,8}$ Thus, to use a lower-frequency
FUS system safely, we need to better understand the parameters that affect the development of cavitation and the information that we can use to detect cavitation formation.

In the current study, we investigated the possibility of inducing thermal lesions in a live pig model by using a 220$\mathrm{kHz}$ MRgFUS system with integrated cavitation detectors. The device operates at the lowest frequency envisioned for brain treatment. Cavitation at this frequency is so likely to occur that it is unknown if it can be avoided during MRgFUS thermal ablation. ${ }^{39,49}$ We aimed to create thermal lesions by varying the acoustic power and duration. We hypothesized that a certain treatment safety threshold exists and that below this threshold the probability of producing inertial cavitation-induced hemorrhagic lesion will be low. We also hypothesized that inertial cavitation activity as measured by passive cavitation detectors will signal the onset of inertial cavitation and correlate with the extent of observed pathological findings.

\section{Methods}

The overall objective of the procedure was to test 2 different types of sonications. FUS-induced temperature elevation in tissues is proportional to the acoustical energy, ${ }^{11}$ that is, proportional to the product of the sonication duration and the acoustical power. The study was thus divided into 2 groups that received either long-duration (40-second) or short-duration (20-second) treatment. The power was adjusted accordingly to induce the same temperature elevation, and the groups are referred to here as the lowpower/long-duration (40-second sonication) and highpower/short-duration (20-second sonication) groups. For each group, the acoustical energy varied from $6 \mathrm{~kJ}$ to 13 $\mathrm{kJ}$. Theoretically, cavitation is difficult to avoid with highpower/short-duration sonication. Thermal rises induced by 20 -second sonications are expected to be more difficult to control because of probabilistic cavitation-enhanced heating. Thermal rises induced by 40 -second sonications are expected to be more controlled if cavitation can be avoided.

All animal work was conducted in accordance with the University of Virginia Animal Care and Use Committee institutional guidelines. The study was approved by the University of Virginia Institutional Review Board. The goal of the current study was not to solve the sonication aberration of ultrasound passing the irregular piglet skull. . $^{34,60}$ Thus, craniectomies were performed in this study.

\section{Neurosurgical Technique}

Ten female piglets (Sus scrofa domesticus) weighing approximately 25-30 lbs underwent general anesthesia with isoflurane inhalation and intubation. A bilateral craniectomy with an average diameter of $3 \mathrm{~cm}$ was performed with a U-shaped incision. With a high-speed electric drill and rongeurs, a craniectomy was performed, leaving the dura intact. Special attention was paid to ensure that the frontal edge of bone flap was away from the frontal sinus, which is located beyond an imaginary line between the eyes of a piglet. The craniectomy site was irrigated with 
large amounts of normal saline to ensure that no residual air bubbles or bone dust remained in the field, because they would significantly degrade the acoustic window through absorption and reflection of the transmitted ultrasound. Subsequently, the scalp was closed with sutures, the subgaleal air was aspirated, and the epidural space was filled with normal saline. The animals were maintained for 1 week before the experiment to allow any remaining air under the skin flap to dissipate.

\section{Magnetic Resonance-Guided Sonication}

Sonications were performed with an ExAblate 4000 Neuro (InSightec), which comprises 1024 ultrasoundtransmitting piezoelectric elements operating at a nominal frequency of $220 \mathrm{kHz}$. The hemispherical geometry of the transducer array is designed for a human-sized skull. The cavitation-detection device is integrated with the FUS transducer. The sonication procedures are described in more detail in the Appendix. All the piglets were intubated and received general anesthesia with isoflurane and ketamine during the sonication procedures, including pre- and postsonication imaging. The piglets' scalps were meticulously shaved in an attempt to avoid air bubbles trapped in the hair. Each animal was placed in a supine position and its normal core body temperature was maintained using a heated circulating water blanket. Each piglet's head was submerged in the circulating predegassed water (dissolved gas, $<1$ parts per million) at a temperature of $15^{\circ}-20^{\circ} \mathrm{C}$. The water level was aligned with the piglet's eyes.

Before sonication, a clinical 3.0-T imaging MRI system (Discovery MR75-3.0T, GE Medical Systems), situated within the FUS treatment room, was employed to ensure that there were no intracranial air bubbles present and to allow for target visualization. Pre- and postsonication MRI protocols included T1-weighted pre- and post-Gd contrast-enhanced, T2-weighted, T2*-weighted, gradientrecalled echo, and FLAIR sequences. A purpose-built 6-channel surface coil (Highfield, LLC) was used to improve the signal-to-noise ratio of the images. The circulation of degassed water was paused during the MRI. All the acquired MRI studies were evaluated by a neuroradiologist (P.R.).

In each experiment, the right-sided ventrolateral thalamus was first treated with low-power/long-duration sonication, and then the left ventrolateral thalamus was treated with high-power/short-duration sonication. Long-duration sonications were performed with a power ranging from 140 to $300 \mathrm{~W}$, and short-duration power ranged from 300 to $700 \mathrm{~W}$ (Table 1).

\section{Cavitation Detection}

For each sonication, back-scattered ultrasound echoes were collected by 2 integrated cavitation detectors manufactured by InSightec. Signals ranging from 50 to $250 \mathrm{kHz}$ were extracted and stored in memory.

For the post-FUS imaging, at least 30 minutes after the completion of sonications, all animals were imaged using the same MRI protocols that were used before the sonications.

\section{Histopathology}

All the animals were killed at the end of the sonication day, and their brains were immediately harvested. The brains were maintained in $10 \%$ formalin solution for at least 10 days after removal. Histological assessment was conducted by a neuropathologist (M.B.L.). The harvested piglet brains underwent a coronal sectioning based on the presentation of lesions on the postprocedure MRI. H \& E staining was conducted to investigate these paraffinembedded, $5-\mu \mathrm{m}$-thick brain sections.

\section{Statistical Analysis}

Continuous data, including the temperatures generated on the MRI thermometry, are presented as mean \pm SD. Those lesions in which a hemorrhage was identified on corresponding MRI and confirmed histologically were classified as being in the hemorrhage group, whereas those with no hemorrhage visualized were classified as being in the nonhemorrhage group. The Student t-test was used to compare the cavitation dose $(\mathrm{V} \cdot \mathrm{Hz})$ difference between the hemorrhage and nonhemorrhage groups. A linear regression model was computed to examine the correlation between the areas of intracranial hemorrhage and cavitation activity. IBM SPSS 20 was used for all statistical studies. All the studies were 2 sided. Results were considered to be statistically significant if the associated $\mathrm{p}$ value was less than 0.05 .

\section{Results}

The left-sided thalamus in Pig 1 was not successfully sonicated because of a hardware failure; therefore, no cavitation information was collected. The peak temperature for Pig 5 was not available for the high-power/short-duration sonication because a large inertial cavitation-induced hemorrhage formed and a large amount of gas was present that affected MRI temperature mapping.

\section{Histopathology}

By varying the sonication parameters, 2 different types of lesions were induced and identified grossly and microscopically (Figs. 1 and 2). Thermal lesions were created in the right thalamus. These lesions were small, soft, and well demarcated and did not show evidence of hemorrhage. Microscopically, an acute ischemic infarct could be identified and characterized by ischemic changes of thalamic neurons and a mild degree of edema (Fig. 1C to E). Mechanical lesions secondary to the inertial cavitation were created on the contralateral (left) thalamus. Five of 9 of these lesions were associated with a severe and poorly localized hemorrhage in the deep gray nuclei, associated with destruction of the parenchyma, ischemic changes, and intense edema (Fig. 2C-E). Figures 1 and 2 illustrate the typical pictures (macro- and microscopic findings, and their corresponding MR images) and descriptions of the 2 different types of lesions.

Thus, low-power, long-duration (40-second) sonications induced thermal lesions, whereas high-power, shortduration (20-second) sonications induced hemorrhagic mechanical lesions. For long-duration sonications, thermal lesions were confirmed by microhistology every time the 
TABLE 1. Thermal lesioning and inertial cavitation-induced mechanical lesioning parameters

\begin{tabular}{|c|c|c|c|c|c|c|c|c|}
\hline \multirow[b]{2}{*}{ Piglet No. } & \multicolumn{4}{|c|}{ Thermal Lesion (right thalamus) } & \multicolumn{4}{|c|}{ Mechanical Lesion (left thalamus) } \\
\hline & Power (W) & Duration (sec) & Energy $(\mathrm{J})$ & Peak Temp $\left({ }^{\circ} \mathrm{C}\right)$ & Power (W) & Duration (sec) & Energy $(\mathrm{J})$ & Peak Temp $\left({ }^{\circ} \mathrm{C}\right)$ \\
\hline 1 & 160 & 40 & 6400 & 55 & - & - & - & - \\
\hline 2 & 140 & 40 & 5600 & 50 & 700 & 20 & 14,000 & 50 \\
\hline 3 & 260 & 40 & 10,400 & 52 & 600 & 20 & 12,000 & 50 \\
\hline 4 & 240 & 40 & 9600 & 55 & 600 & 20 & 12,000 & 53 \\
\hline 5 & 260 & 40 & 10,400 & 59 & 300 & 20 & 6000 & $N A^{*}$ \\
\hline 6 & 150 & 40 & 6000 & 53 & 400 & 20 & 8000 & 56 \\
\hline 7 & 300 & 40 & 12,000 & 56 & 400 & 20 & 8000 & 57 \\
\hline 8 & 234 & 40 & 9360 & 55 & 300 & 20 & 6000 & 56 \\
\hline 9 & 220 & 40 & 8800 & 53 & 400 & 20 & 8000 & 54 \\
\hline 10 & 270 & 40 & 10,800 & 49 & 450 & 20 & 9000 & 54 \\
\hline
\end{tabular}

$\mathrm{NA}=$ not available; Temp $=$ temperature.

* Data not available. The focal temperature in that lesion measured on MR thermometry was not achieved because of a large intracranial hemorrhage extending to the left lateral ventricle resulting from the sonication ( $300 \mathrm{~W}$ for 20 seconds). It should be noted that 20 seconds was the setting for treatment duration. The actual cavitation occurred earlier.

peak temperature was higher than $55^{\circ} \mathrm{C}$, and no instance of a thermal lesion was found for peak temperatures lower than $53^{\circ} \mathrm{C}$ (Table 2).

\section{Magnetic Resonance Imaging}

Thermal and mechanical lesions started to present on
T2-weighted MRI approximately 30 minutes after the ablation (Table 2). Thermal lesions were located in the right thalamus, with a hypo- or isointensity on T1-weighted imaging, a rimlike hyperintensity on T2-weighted imaging (Fig. 1B), and isointensity on T1-weighted gradientrecalled echo imaging. The location of the lesion on MRI
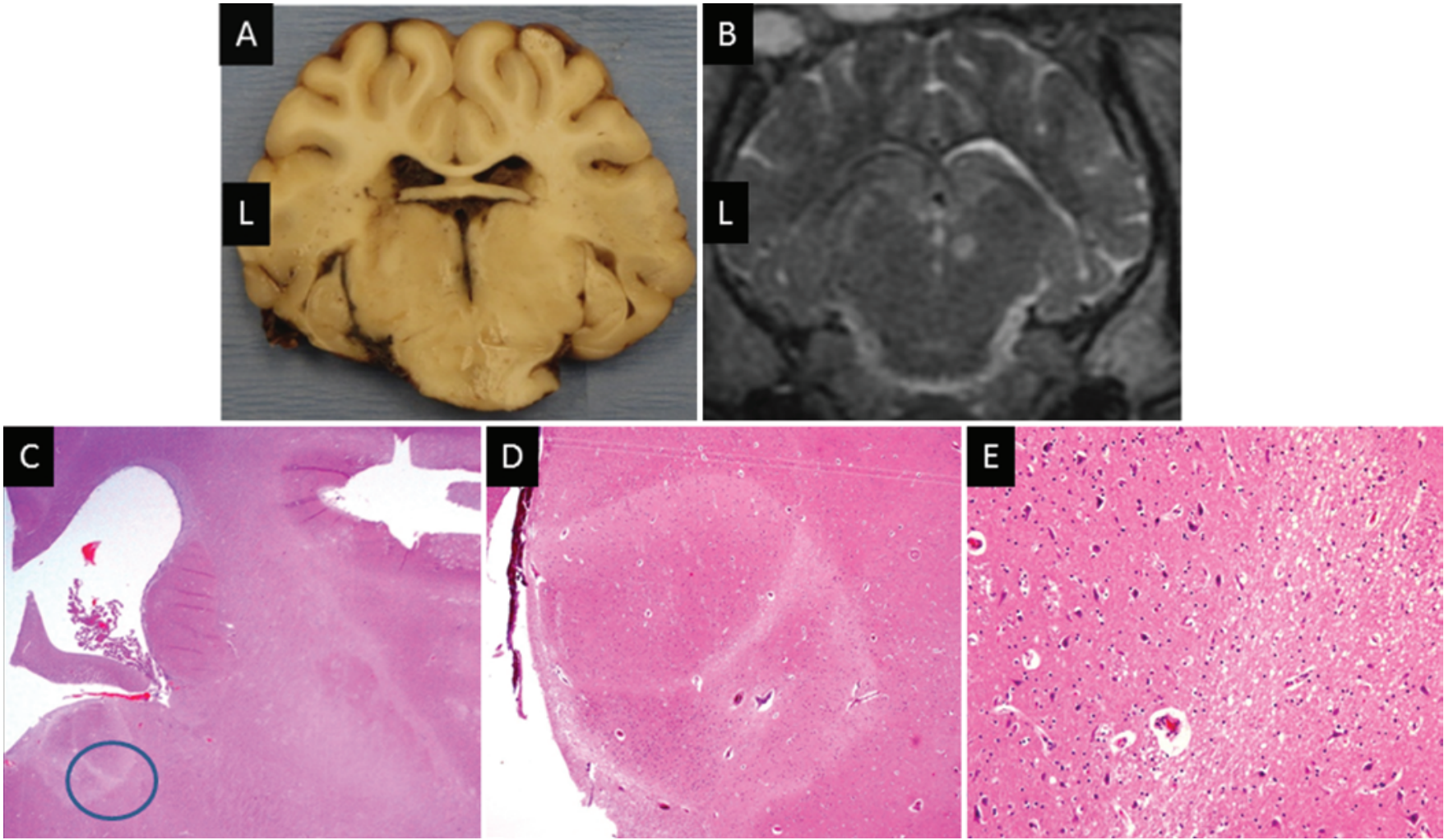

FIG. 1. A-E: Photograph of specimen, MR image, and photomicrographs of the thermal lesion created in Pig 4 using ultrasound parameters of $240 \mathrm{~W}$ for 40 seconds. A well-demarcated acute infarction characterized by a central ischemic necrosis and surrounding edema, not associated with inflammatory reaction, can be seen. C and D: Right side of the brain showing a well-delineated acute ischemic infarct with minimal surrounding edema. E: Higher magnification of the lesion (circled in C) shows edema, early coagulative necrosis, and hypereosinophilic neurons consistent with an acute infarction. $\mathrm{H} \& \mathrm{E}$, original magnification $\times 20$ $(C), \times 40(D)$, and $\times 100(E) . L=$ left side. Figure is available in color online only. 

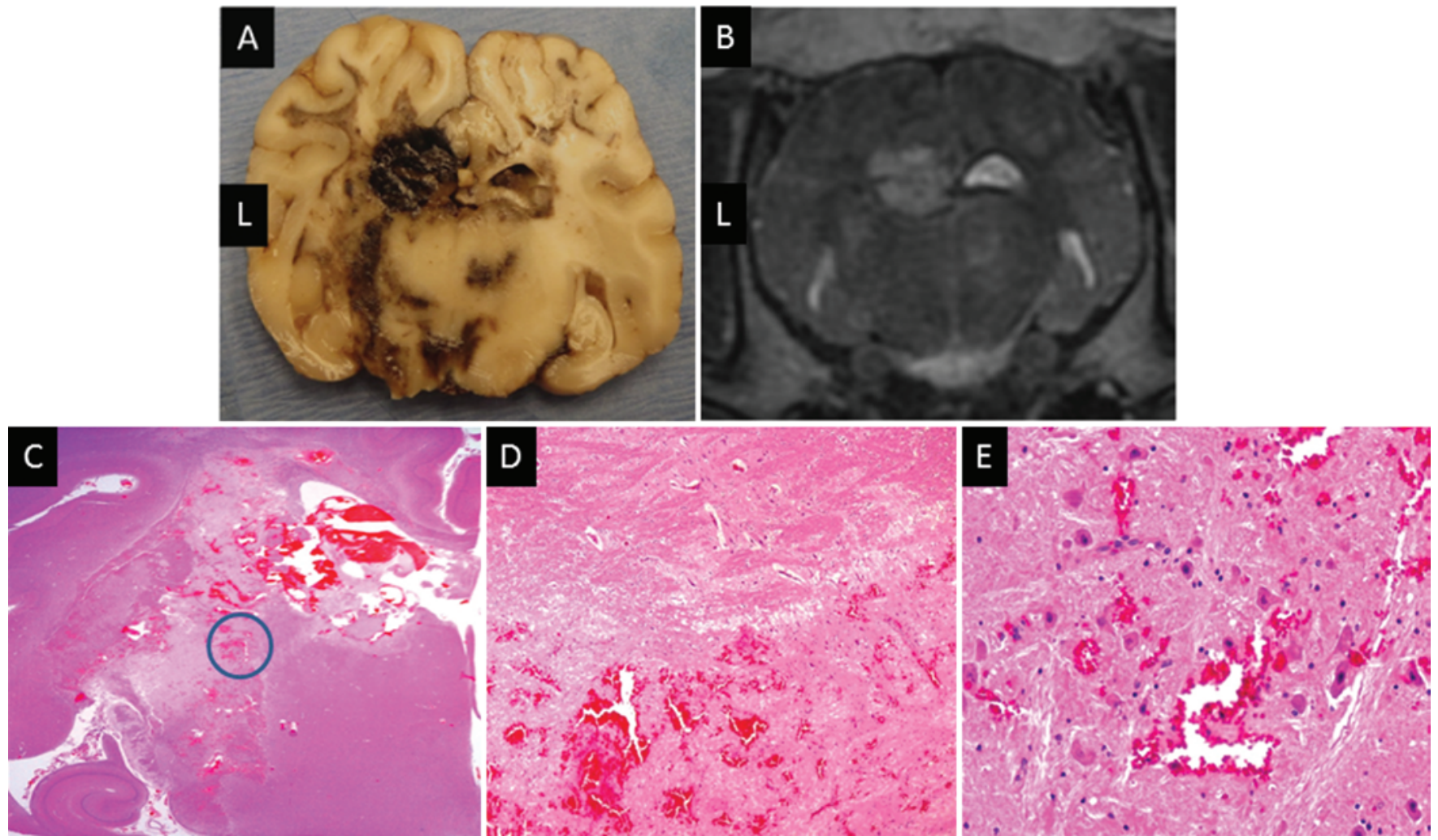

FIG. 2. A-E: Photograph of specimen, MR image, and photomicrographs of the inertial cavitation-induced mechanical lesion. An inertial cavitation-induced mechanical lesion (A-E) formed in Pig 5 using $300 \mathrm{~W}$ for 20 seconds. A large intracranial hemorrhagic infarct was generated with extension to the left lateral ventricle. C: Left side of the brain showing a large acute hemorrhage in the area of the thalamus with extension into the lateral ventricle. D-E: Higher magnification of the lesion (circled in C) shows significant edema, early coagulative necrosis, multifocal hemorrhage, and hypereosinophilic neurons consistent with an acute infarction. $\mathrm{H} \& \mathrm{E}$, original magnification $\times 20(\mathrm{C}), \times 40(\mathrm{D})$, and $\times 200(\mathrm{E}) . \mathrm{L}=$ left side. Figure is available in color online only.

corresponded to the approximate position of the target on the treatment plan. After the administration of Gd, 1 thermal lesion across all animals produced a ringlike contrast enhancement $(\mathrm{n}=1)$, whereas the remainder of the thermal lesions were not contrast enhancing $(n=9)$.

An example of an inertial cavitation-induced lesion generated on the left side of the thalamus can be seen in Fig. 2A, which shows a distinct lesion with hyperintensity manifested on T2-weighted MRI approximately 30 minutes after the sonication. The maximum dimensions of the hemorrhagic lesions varied between 3.8 and $12.6 \mathrm{~mm}$.

\section{Cavitation Detection}

Typical spectra recorded during sonications are displayed in Fig. 3. In the absence of acoustic cavitation activity, a sole peak at the central frequency of $231 \mathrm{kHz}$ was observed (Fig. 3A), corresponding to the backscattered echoes of the emitted signals. In the presence of cavitation bubbles, however, subharmonic acoustic emissions appear in the detector signals. ${ }^{30}$ The manifestation of a sharp, isolated peak at a half of the fundamental frequency is the signature of stable cavitation $\left(116 \mathrm{kHz}\right.$; Fig. 3B).$^{55}$ Detected inertial cavitation appeared as broadband noise emission and broadened shoulders (ranging from 50 to $180 \mathrm{kHz}$ ) around the half harmonic peak, ${ }^{17}$ resulting from the collapse of the cavitation bubbles (Fig. 3C). Inertial cavitation was elicited on the left thalamus in all but Pig 1 .

Because the occurrence of inertial cavitation is known to induce intracranial hemorrhage, ${ }^{21}$ we further processed the cavitation spectra to quantify the amount of cavitation activity occurring during the sonications. Each spectrum was integrated over the 50 - to $180-\mathrm{kHz}$ frequency range. This value is hereafter referred to as the cavitation dose. The maximum cavitation dose recorded during sonication was plotted against the status of a hemorrhage identified on MRI and gross findings. The cavitation doses for sonications that did not result in hemorrhage were plotted in the left column, and the sonications associated with hemorrhage were plotted in the right column, which led to a natural divide in the data at a value of approximately 0.09 $\mathrm{V} \cdot \mathrm{Hz}$ (Fig. 4). Cavitation doses that were not associated with the occurrence of a hemorrhage were gathered below the threshold of $0.09 \mathrm{~V} \cdot \mathrm{Hz}$, whereas the doses associated with a hemorrhage were above that value.

In addition, the elliptical surface area of each hemorrhage was determined on the macroscopic histology using ImageJ software (http://rsbweb.nih.gov/ij/download.html). The largest dimension of hemorrhage was then plotted against the maximum cavitation dose recorded during the treatment of the given hemisphere using a linear regression model (coefficient of determination $\left[\mathrm{R}^{2}\right]=0.74$; 
TABLE 2. Thermal lesions and inertial cavitation-induced mechanical lesions in thalami and corresponding cavitation-induced hemorrhage

\begin{tabular}{|c|c|c|c|c|c|c|c|c|}
\hline \multirow[b]{2}{*}{ Piglet No. } & \multicolumn{4}{|c|}{ Thermal Lesion (right thalamus) } & \multicolumn{4}{|c|}{ Mechanical (hemorrhagic) Lesion (left thalamus) } \\
\hline & Peak Temp $\left({ }^{\circ} \mathrm{C}\right)$ & MRI & Macroscopic & Microscopic & Peak Temp $\left({ }^{\circ} \mathrm{C}\right)$ & MRI & Macroscopic & Microscopic \\
\hline $1^{*}$ & 55 & Y & Y & Y & - & - & - & - \\
\hline 2 & 50 & $\mathrm{~N}$ & $\mathrm{~N}$ & $\mathrm{~N}$ & 50 & $\mathrm{~N}$ & $\mathrm{~N}$ & $\mathrm{~N}$ \\
\hline 3 & 52 & $\mathrm{~N}$ & $\mathrm{~N}$ & $\mathrm{~N}$ & 50 & $\mathrm{~N}$ & $\mathrm{~N}$ & $\mathrm{~N}$ \\
\hline 4 & 55 & Y & $\mathrm{N}$ & Y & 63 & $Y$ & $Y$ & $Y$ \\
\hline $5 \dagger$ & 59 & $Y$ & Y & Y & NA & $Y$ & $Y$ & $Y$ \\
\hline 6 & 53 & $\mathrm{~N}$ & $\mathrm{~N}$ & $\mathrm{~N}$ & 56 & $Y$ & $Y$ & $Y$ \\
\hline 7 & 56 & $Y$ & $Y$ & $Y$ & 57 & $Y$ & $Y$ & $Y$ \\
\hline 8 & 55 & $Y$ & $Y$ & $Y$ & 56 & $Y$ & $Y$ & $Y$ \\
\hline 9 & 53 & $Y$ & $\mathrm{~N}$ & $\mathrm{~N}$ & 54 & $\mathrm{~N}$ & $\mathrm{~N}$ & $\mathrm{~N}$ \\
\hline 10 & 49 & $\mathrm{~N}$ & $\mathrm{~N}$ & $\mathrm{~N}$ & 54 & $\mathrm{~N}$ & $\mathrm{~N}$ & $\mathrm{~N}$ \\
\hline \multicolumn{9}{|c|}{$\begin{array}{l}Y=\text { detectable; } N=\text { not detectable. } \\
\text { * FUS was not performed successfully on the left thalamus in Pig } 1 \text { because of a hardware glitch. } \\
\dagger \text { The MRI temperature in Pig } 5 \text { was not achievable because a large intracranial hemorrhage extending to the left lateral ventricle was gener- } \\
\text { ated as a result of the sonication ( } 300 \mathrm{~W} \text { for } 20 \text { seconds). The thermal lesion was an acute infarct, while the mechanical lesion was an acute } \\
\text { hemorrhagic infarct. }\end{array}$} \\
\hline
\end{tabular}

Fig. 5). The $\mathrm{R}^{2}$ value for this plot was 0.7435 , indicating a moderately strong correlation between cavitation-induced hemorrhage size and maximum cavitation dose.

\section{Discussion}

The ultrasonic aberration induced by the human skull decreases with decreasing frequency, making it easier to deliver FUS to the brain transcranially. Nevertheless, decreasing the frequency increases the probability of cavitation and of hemorrhage. The need for precise transcranial adaptive focusing techniques for an efficient treatment is relaxed, but the need for a means of ensuring cavitation safety becomes paramount. Thus, low-frequency brain treatments should be used only after thorough safety testing has been completed.

\section{Lesion Formation}

The objective of the current study was to demonstrate that thermal lesions can be induced with a $220-\mathrm{kHz}$ MRgFUS system in a preclinical in vivo animal model and to explore the correlation between detected acoustic inertial cavitation and any induced intracranial hemorrhage (or other unintended tissue damage). Thermal lesions have been successfully induced at the spot where the peak voxel temperature reached $55^{\circ} \mathrm{C}$ or more during the 40 -second sonication time. In general, the identified lesions (whether thermal or mechanical) were oval, with the

\section{A No Cavitation}

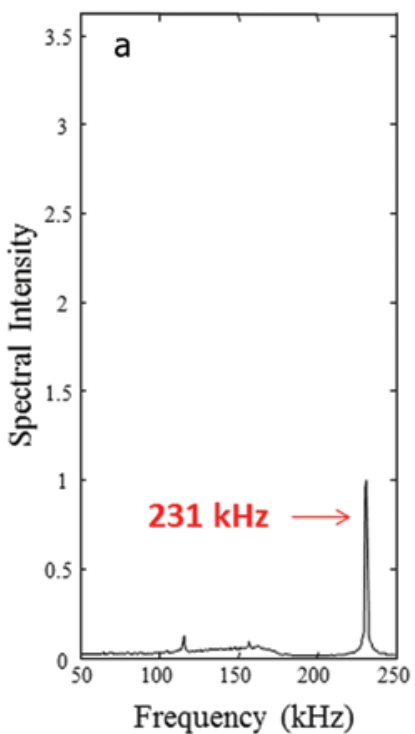

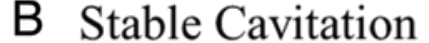

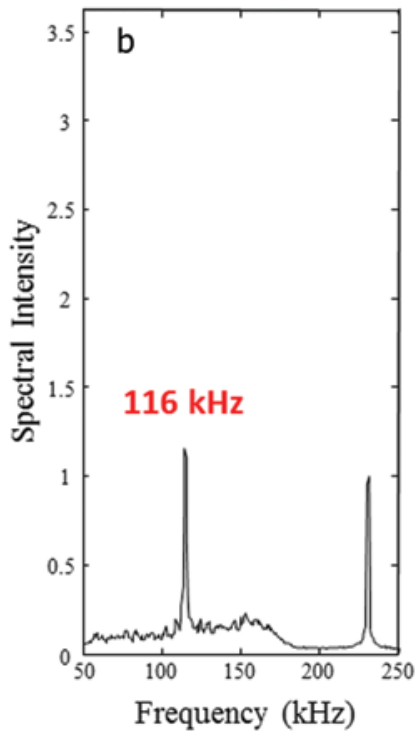

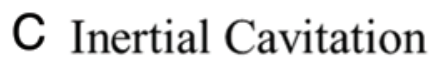

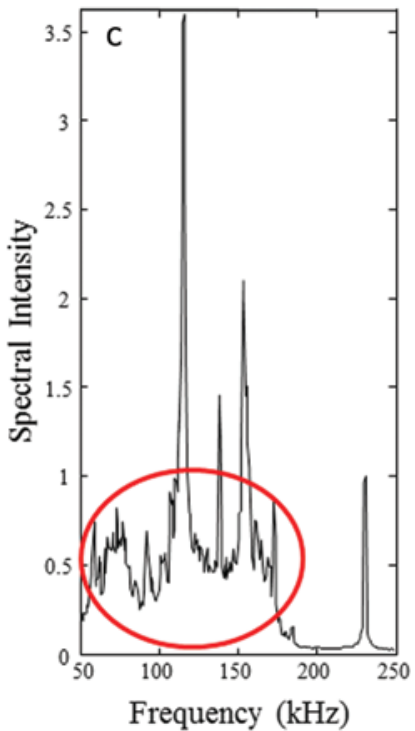

FIG. 3. A-C: Schematic of acoustic emission spectra indicating patterns of inertial cavitation-related changes. Figure is available in color online only. 


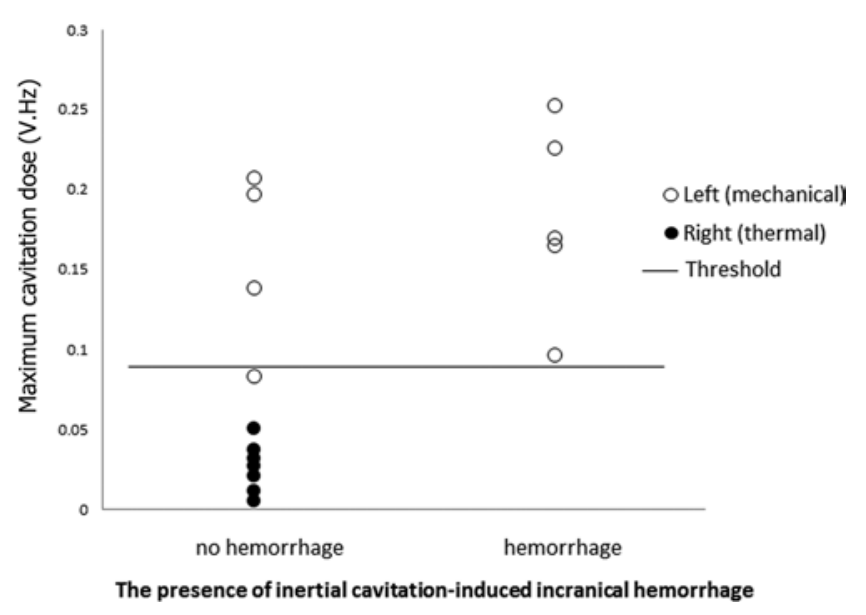

FIG. 4. Identification of the inertial cavitation threshold in relation to the intracranial hemorrhage in the thermal and mechanical lesions.

major axis parallel to the transducer-focus direction. In the current study, the relative temperature calculated on the basis of MR thermometry indicated that a thermal lesion was generated once the peak temperature was higher than $55^{\circ} \mathrm{C}$; otherwise, no lesion was detected either on MRI or on macroscopic histological assessment. Cohen et al. explored the feasibility of using the same animal model to prove the success of creating a thermal lesion in the live pig brain with a high-frequency (1.15-MHz) device and found that the temperature threshold was approximately $60^{\circ} \mathrm{C}$, which is slightly higher than our finding. ${ }^{5}$ It is known that tissue damage is best assessed by the thermal dose introduced by Sapareto and Dewey. ${ }^{52}$ The thermal dose is the integral of the thermal rise as a function of time. The same thermal dose can be achieved with a high thermal rise and low sonication duration or with a lower thermal rise but a longer sonication duration. Cohen et al. used a shorter sonication duration (between 12 and 16 seconds) than we did in our study (20-40 seconds). Thus, their higher temperature threshold is in line with that of our study. Nevertheless, the 20-second sonication time for the high-power/short-duration sonications induced hemorrhages in $5(56 \%)$ of 9 pigs with inertial cavitation. It is fortunate that our study demonstrates the existence of a cavitation dose threshold below which the risk of intracranial hemorrhage seems low.

\section{Limitations of the Piglet Model}

There are several features that make the piglet brain an effective model for FUS research. First, it is anatomically similar to the human brain, although the interior layer of the skull is irregular, and the olfactory bulb is much larger. Second, piglets are more available and less expensive to maintain than nonhuman primates. Third, the size of their brains is similar to that of other large animals. Hence, piglets have been widely used for in vivo studies of FUS..$^{5,13,43}$

However, the use of piglets is not without drawbacks. The low-frequency MRgFUS device is more apt to create intracranial and extracranial air bubbles than higherfrequency devices. Therefore, a minimum of 1 week of recovery time after craniectomy was allowed for the complete absorption of any potential intracranial bubbles.

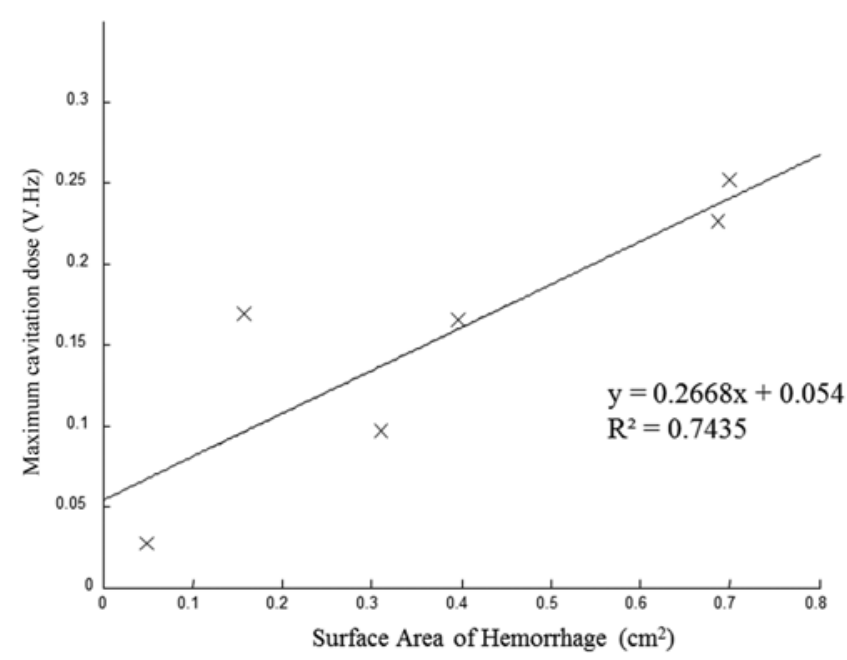

FIG. 5. Correlation between the hemorrhage size on macroscopic histopathological study and acoustic signal intensity.

Sonication with the low-frequency system was not achievable without severe scalp burning unless the scalp hair was completely shaved (data not shown). The amplitude apodization rate of $83 \%$ in the current study is appropriate for ensuring that all acoustic beams pass through the craniectomy and to eliminate the risk of skull heating and penetration of the frontal sinus. Although the planning system provides for manual specification of no-pass regions, using a predefined circular apodization pattern saved considerable planning time and ensured that the same aperture was used for all the experiments. Visual inspection was performed to double-check the efficacy of using apodization to block ultrasound, as allowed by the planning software of the device.

In preclinical rodent models, large aperture arrays such as that used in this experiment do not require aberration correction, given the thickness of the skull bone. ${ }^{10}$ However, the flat superior and irregular inner surfaces of the piglet skull make it extremely difficult to achieve consistent sonication results with our device without the use of a craniectomy as an acoustic window, which is why we chose a craniotomy procedure for our experiment. It would be difficult to perform the same experiments in noncraniectomized piglets without changing the ultrasonic transducer; the translation of such results to the clinical system would not be straightforward. A more realistic follow-up study would involve using the same animal model with an ex vivo human calvaria inserted between the animal's brain and the preclinical transducer used in our study.

\section{Comparison With Existing Systems}

Most brain systems designed for tissue ablation in humans operate at a higher frequency, ranging from 0.66 $\mathrm{MHz}^{12,26,31}$ to $1 \mathrm{MHz}$.,33 They afford an exceedingly lower risk of inertial cavitation-induced hemorrhage than the $0.22-\mathrm{MHz}$ system used in this study and instead induce purely thermal lesions. The $0.6-\mathrm{MHz}$ device has shown promising clinical results for neuropathic pain ${ }^{26,45}$ and essential tremor ${ }^{12,33}$ and holds promise for treating epilepsy ${ }^{42}$ and trigeminal neuralgia ${ }^{44}$ and for performing cinguloto- 
my to alleviate pain, depression, and obsessive compulsive disorders ${ }^{42}$ or for localized and transient opening of the blood-brain barrier. ${ }^{25}$ Other low-frequency devices were developed recently to take advantage of the mechanical, rather than the thermal, effects of ultrasound and expand the treatment envelope. McDannold et al. performed nonthermal ablation with microbubble-enhanced FUS close to the optic tract. ${ }^{40}$ Shimizu et al. investigated the safety of transcranial low-frequency (0.1- to $1-\mathrm{MHz})$ ultrasonic thrombolysis for acute ischemic stroke and assessed the safety of a $0.49-\mathrm{MHz}$ device for sonothrombolysis in a primate model..$^{54}$ Low-frequency, low-intensity devices have also been designed for ultrasonic neuromodulation in humans. Neuromodulation has been achieved in vivo in primates ${ }^{9}$ with a $0.32-\mathrm{MHz}$ transducer ${ }^{61}$ and in humans ${ }^{29}$ with a $0.5-\mathrm{MHz}$ transducer. Neuromodulation was achieved with acoustic intensities at foci of $4 \mathrm{~W} / \mathrm{cm}^{2}$ and $5.9 \mathrm{~W} /$ $\mathrm{cm}^{2}$ in primates ${ }^{9}$ and humans, respectively. ${ }^{29}$ No adverse effects were reported in either of these studies. At such intensities, cavitation events like the ones presented in this study are not likely to occur. Nevertheless, intramembrane microcavitation has been hypothesized as a possible mechanism for explaining acoustical triggering of action potentials. ${ }^{28,50}$

\section{Cavitation Detection}

Previous passive cavitation detection studies focused largely on the use of ultrasound in conjunction with microbubble injection. ${ }^{20,37,57}$ Studies of inertial cavitation using low-frequency FUS in large animals are largely lacking. In vivo cavitation detection in the brain of a large animal was performed recently at $660 \mathrm{kHz}$, with a reported peak negative cavitation threshold of $11 \mathrm{MPa}^{16}$ Nevertheless, the study was conducted with a very short sonication time (several microseconds) to avoid thermal rise. Our study provides, to our knowledge, the first data of in vivo cavitation activity in the brain with low-frequency ultrasound and therapeutic ultrasonic parameters, showing an estimate of a lower threshold of $0.09 \mathrm{~V} \cdot \mathrm{Hz}$ for the development of inertial cavitation with our specific cavitation-detection setup. In the "no hemorrhage" column in Fig. 4, several points lay above the threshold line. For these events, according to the spectra, acoustic cavitation occurred, but it might have taken place somewhere outside the brain, such as in the skin or within the water bath in front of the transducer. Our results suggest that a lower threshold for inertial cavitation exists; however, more data are required to confirm and refine the precision of that threshold. In the future, the use of multiple cavitation detectors could be envisioned to ultrasonically map cavitation activity and avoid taking into account cavitation occurring outside the brain. ${ }^{27}$ Ideally, if a firm threshold for inertial cavitation can be determined, it can be built into clinical FUS devices as a safety interlock that triggers a closed-loop power-modulation scheme to minimize the risk of cavitationinduced intracranial hemorrhage.

\section{Study Limitations}

This study was performed in 10 piglet brains, and such a small sample size limits the power of the study. After adjustment of the built-in passive cavitation-detection de- vice based on our findings, additional preclinical studies will need to be conducted to investigate and confirm the threshold of inertial cavitation, particularly in animals with an intact cranium. This study also points out the narrow boundary between thermal and mechanical effects in terms of acoustical parameters at low frequency; $260 \mathrm{~W}$ for 40 seconds has been shown to induce a focal thermal lesion, whereas $300 \mathrm{~W}$ for 20 seconds has been shown to induce massive hemorrhage. This stresses the importance of cavitation monitoring and safety when working at low frequencies. Nevertheless, cavitation activity can be monitored and real-time closed-loop control can help shut down the ultrasound as soon as the first microbubbles are formed and before they coalesce into larger bubbles that can induce hemorrhage when they collapse.

\section{Conclusions}

To our knowledge, this is the first in vivo brain study to demonstrate the likelihood of detection of a cavitation threshold secondary to certain power levels and sonication durations used with a low-frequency MRgFUS system. A thermal ablation lesion with ischemic changes was achieved with the $220-\mathrm{kHz}$ FUS system by selecting the appropriate sonication parameters. The cavitation threshold identified herein may provide extremely important information for the avoidance of inertial cavitation-induced tissue damage with low-frequency ultrasound.

\section{Appendix \\ Sonication Procedure}

The sonication procedure aimed at minimizing the total number of ultrasonic sonications at a given target. The temperature elevation induced at focus depends on the type of tissue (gray and white matter have different acoustical absorption coefficients) and on the perfusion rate (that acts as a heat sink). This is why the same acoustical power can induce different thermal rises in 2 different animals. Fortunately, the thermal rise can be measured with dedicated MRI temperature mapping. Verification of sonication with a low power ( $20 \mathrm{~W}$ for 20 seconds) was first initiated, which allowed for $1^{\circ}-2^{\circ} \mathrm{C}$ of temperature rise on the MR thermometry to confirm accuracy of the sonication location. Once the targeting thalamus location was identified, a slightly higher energy sonication was performed (50-W power, 40-second duration). The corresponding temperature elevation $(\Delta T)$ was measured, and the power was then prorated to target $58^{\circ} \mathrm{C}$ according to the following equation: power $=50(58-37) / \Delta T$. Thus, a total of 3 sonications were performed at each location. The first 2 sonications were identical for all locations, and the power of the third one (the only one at therapeutic power) is listed in Table 1. All of the pig sonications were delivered in continuous-wave mode. The cooling time between sonications was provided by the system.

\section{Additional Information About the Transducer}

The transducer used in this study was $300 \mathrm{~mm}$ in diameter and curved at a radius of $150 \mathrm{~mm}$, with an emitting surface of 1413.7 $\mathrm{cm}^{2}$. For all sonications, a circumferential ring of transducer elements was disabled to produce an apodization rate of $83 \%$ to ensure that the transmitted ultrasound beams would pass through the craniectomy.

\section{Cavitation Detectors}

The detectors are undamped air-backed elements with a single frontal matching layer (manufactured by InSightec). The useful fre- 
quency response of the detectors ranged from 50 to $250 \mathrm{kHz}$. The frequency spectrum within this bandwidth was stored in memory after Fourier transformation of the recorded signals.

\section{Acknowledgments}

We are grateful to Ms. Brenna Beckelman for the assistance in surgery preparation and brain harvesting. We also appreciate the excellent veterinary care and assistance from Jeremy Gatesman and Gina Wimer.

\section{References}

1. Arvanitis CD, Livingstone MS, McDannold N: Combined ultrasound and MR imaging to guide focused ultrasound therapies in the brain. Phys Med Biol 58:4749-4761, 2013

2. Baron C, Aubry JF, Tanter M, Meairs S, Fink M: Simulation of intracranial acoustic fields in clinical trials of sonothrombolysis. Ultrasound Med Biol 35:1148-1158, 2009

3. Behrens S, Daffertshofer M, Spiegel D, Hennerici M: Lowfrequency, low-intensity ultrasound accelerates thrombolysis through the skull. Ultrasound Med Biol 25:269-273, 1999

4. Chauvet D, Marsac L, Pernot M, Boch AL, Guillevin R, Salameh N, et al: Targeting accuracy of transcranial magnetic resonance-guided high-intensity focused ultrasound brain therapy: a fresh cadaver model. Laboratory investigation. J Neurosurg 118:1046-1052, 2013

5. Cohen ZR, Zaubermann J, Harnof S, Mardor Y, Nass D, Zadicario E, et al: Magnetic resonance imaging-guided focused ultrasound for thermal ablation in the brain: a feasibility study in a swine model. Neurosurgery 60:593-600, 2007

6. Crum LA: Cavitation microjets as a contributory mechanism for renal calculi disintegration in ESWL. J Urol 140:15871590,1988

7. Daffertshofer M, Fatar M: Therapeutic ultrasound in ischemic stroke treatment: experimental evidence. Eur J Ultrasound 16:121-130, 2002

8. Daffertshofer M, Gass A, Ringleb P, Sitzer M, Sliwka U, Els $\mathrm{T}$, et al: Transcranial low-frequency ultrasound-mediated thrombolysis in brain ischemia: increased risk of hemorrhage with combined ultrasound and tissue plasminogen activator: results of a phase II clinical trial. Stroke 36:1441-1446, 2005

9. Deffieux T, Younan Y, Wattiez N, Tanter M, Pouget P, Aubry JF: Low-intensity focused ultrasound modulates monkey visuomotor behavior. Curr Biol 23:2430-2433, 2013

10. Dervishi E, Larrat B, Pernot M, Adam C, Marie Y, Fink M, et al: Transcranial high intensity focused ultrasound therapy guided by 7 TESLA MRI in a rat brain tumour model: a feasibility study. Int J Hyperthermia 29:598-608, 2013

11. Eames MDC, Hananel A, Snell JW, Kassell NF, Aubry JF: Trans-cranial focused ultrasound without hair shaving: feasibility study in an ex vivo cadaver model. J Ther Ultrasound 1:24, 2014

12. Elias WJ, Huss D, Voss T, Loomba J, Khaled M, Zadicario E, et al: A pilot study of focused ultrasound thalamotomy for essential tremor. N Engl J Med 369:640-648, 2013

13. Elias WJ, Khaled M, Hilliard JD, Aubry JF, Frysinger RC, Sheehan JP, et al: A magnetic resonance imaging, histological, and dose modeling comparison of focused ultrasound, radiofrequency, and Gamma Knife radiosurgery lesions in swine thalamus. Laboratory investigation. J Neurosurg 119:307-317, 2013

14. Foley JL, Eames M, Snell J, Hananel A, Kassell N, Aubry JF: Image-guided focused ultrasound: state of the technology and the challenges that lie ahead. Imaging Med 5:357-370, 2013

15. Fry FJ, Barger JE: Acoustical properties of the human skull. J Acoust Soc Am 63:1576-1590, 1978
16. Gateau J, Aubry JF, Chauvet D, Boch AL, Fink M, Tanter M: In vivo bubble nucleation probability in sheep brain tissue. Phys Med Biol 56:7001-7015, 2011

17. Gateau J, Aubry JF, Pernot M, Fink M, Tanter M: Combined passive detection and ultrafast active imaging of cavitation events induced by short pulses of high-intensity ultrasound. IEEE Trans Ultrason Ferroelectr Freq Control 58:517532,2011

18. Gateau J, Taccoen N, Tanter M, Aubry JF: Statistics of acoustically induced bubble-nucleation events in in vitro blood: a feasibility study. Ultrasound Med Biol 39:1812-1825, 2013

19. Guthkelch AN, Carter LP, Cassady JR, Hynynen KH, Iacono RP, Johnson PC, et al: Treatment of malignant brain tumors with focused ultrasound hyperthermia and radiation: results of a phase I trial. J Neurooncol 10:271-284, 1991

20. Holt RG, Roy RA: Measurements of bubble-enhanced heating from focused, $\mathrm{MHz}$-frequency ultrasound in a tissuemimicking material. Ultrasound Med Biol 27:1399-1412, 2001

21. Hwang JH, Tu J, Brayman AA, Matula TJ, Crum LA: Correlation between inertial cavitation dose and endothelial cell damage in vivo. Ultrasound Med Biol 32:1611-1619, 2006

22. Hynynen K: MRI-guided focused ultrasound treatments. Ultrasonics 50:221-229, 2010

23. Hynynen K: The threshold for thermally significant cavitation in dog's thigh muscle in vivo. Ultrasound Med Biol 17:157-169, 1991

24. Hynynen K, McDannold N, Vykhodtseva N, Jolesz FA: Noninvasive MR imaging-guided focal opening of the bloodbrain barrier in rabbits. Radiology 220:640-646, 2001

25. Hynynen K, McDannold N, Vykhodtseva N, Raymond S, Weissleder R, Jolesz FA, et al: Focal disruption of the bloodbrain barrier due to $260-\mathrm{kHz}$ ultrasound bursts: a method for molecular imaging and targeted drug delivery. J Neurosurg 105:445-454, 2006

26. Jeanmonod D, Werner B, Morel A, Michels L, Zadicario E, Schiff G, et al: Transcranial magnetic resonance imagingguided focused ultrasound: noninvasive central lateral thalamotomy for chronic neuropathic pain. Neurosurg Focus 32(1):E1, 2012

27. Jones RM, O’Reilly MA, Hynynen K: Transcranial passive acoustic mapping with hemispherical sparse arrays using CT-based skull-specific aberration corrections: a simulation study. Phys Med Biol 58:4981-5005, 2013

28. Krasovitski B, Frenkel V, Shoham S, Kimmel E: Intramembrane cavitation as a unifying mechanism for ultrasound-induced bioeffects. Proc Natl Acad Sci U S A 108:3258-3263, 2011

29. Legon W, Sato TF, Opitz A, Mueller J, Barbour A, Williams A, et al: Transcranial focused ultrasound modulates the activity of primary somatosensory cortex in humans. Nat Neurosci 17:322-329, 2014

30. Leighton TG: The Acoustic Bubble. London: Academic Press, 1994

31. Lipsman N, Schwartz ML, Huang Y, Lee L, Sankar T, Chapman M, et al: MR-guided focused ultrasound thalamotomy for essential tremor: a proof-of-concept study. Lancet Neurol 12:462-468, 2013

32. Lynn JG, Zwemer RL, Chick AJ, Miller AE: A new method for the generation and use of focused ultrasound in experimental biology. J Gen Physiol 26:179-193, 1942

33. Marquet F, Boch AL, Pernot M, Montaldo G, Seilhean D, Fink M, et al: Non-invasive ultrasonic surgery of the brain in non-human primates. J Acoust Soc Am 134:1632-1639, 2013

34. Marquet F, Pernot M, Aubry JF, Montaldo G, Marsac L, Tanter M, et al: Non-invasive transcranial ultrasound therapy based on a 3D CT scan: protocol validation and in vitro results. Phys Med Biol 54:2597-2613, 2009 
35. Marsac L, Chauvet D, Larrat B, Pernot M, Robert B, Fink M, et al: MR-guided adaptive focusing of therapeutic ultrasound beams in the human head. Med Phys 39:1141-1149, 2012

36. Martin E, Jeanmonod D, Morel A, Zadicario E, Werner B: High-intensity focused ultrasound for noninvasive functional neurosurgery. Ann Neurol 66:858-861, 2009

37. McDannold N, Arvanitis CD, Vykhodtseva N, Livingstone MS: Temporary disruption of the blood-brain barrier by use of ultrasound and microbubbles: safety and efficacy evaluation in rhesus macaques. Cancer Res 72:3652-3663, 2012

38. McDannold N, Clement GT, Black P, Jolesz F, Hynynen K: Transcranial magnetic resonance imaging- guided focused ultrasound surgery of brain tumors: initial findings in 3 patients. Neurosurgery 66:323-332, 2010

39. McDannold N, Park EJ, Mei CS, Zadicario E, Jolesz F: Evaluation of three-dimensional temperature distributions produced by a low-frequency transcranial focused ultrasound system within ex vivo human skulls. IEEE Trans Ultrasoun Ferroelectr Freq Control 57:1967-1976, 2010

40. McDannold N, Zhang YZ, Power C, Jolesz F, Vykhodtseva $\mathrm{N}$ : Nonthermal ablation with microbubble-enhanced focused ultrasound close to the optic tract without affecting nerve function. Laboratory investigation. J Neurosurg 119:12081220,2013

41. Miller MW, Miller DL, Brayman AA: A review of in vitro bioeffects of inertial ultrasonic cavitation from a mechanistic perspective. Ultrasound Med Biol 22:1131-1154, 1996

42. Monteith S, Sheehan J, Medel R, Wintermark M, Eames M, Snell J, et al: Potential intracranial applications of magnetic resonance-guided focused ultrasound surgery. A review. J Neurosurg 118:215-221, 2013

43. Monteith SJ, Harnof S, Medel R, Popp B, Wintermark M, Lopes MB, et al: Minimally invasive treatment of intracerebral hemorrhage with magnetic resonance-guided focused ultrasound. J Neurosurg 118:1035-1045, 2013

44. Monteith SJ, Medel R, Kassell NF, Wintermark M, Eames $\mathrm{M}$, Snell J, et al: Transcranial magnetic resonance-guided focused ultrasound surgery for trigeminal neuralgia: a cadaveric and laboratory feasibility study. Laboratory investigation. J Neurosurg 118:319-328, 2013

45. Moser D, Zadicario E, Schiff G, Jeanmonod D: MR-guided focused ultrasound technique in functional neurosurgery: targeting accuracy. J Ther Ultrasound 1:3, 2013

46. Nyborg WL: Biological effects of ultrasound: development of safety guidelines. Part II: general review. Ultrasound Med Biol 27:301-333, 2001

47. Ohl CD, Arora M, Ikink R, de Jong N, Versluis M, Delius M, et al: Sonoporation from jetting cavitation bubbles. Biophys J 91:4285-4295, 2006

48. Pernot M, Aubry JF, Tanter M, Boch AL, Marquet F, Kujas $\mathrm{M}$, et al: In vivo transcranial brain surgery with an ultrasonic time reversal mirror. J Neurosurg 106:1061-1066, 2007

49. Pinton G, Aubry JF, Fink M, Tanter M: Numerical prediction of frequency dependent 3D maps of mechanical index thresholds in ultrasonic brain therapy. Med Phys 39:455-467, 2012

50. Plaksin M, Shoham S, Kimmel E: Intramembrane cavitation as a predictive bio-piezoelectric mechanism for ultrasonic brain stimulation. Physical Review X. (http://journals.aps. org/prx/abstract/10.1103/PhysRevX.4.011004) [Accessed October 1, 2014]
51. Pulkkinen A, Hynynen K: Computational aspects in high intensity ultrasonic surgery planning. Comput Med Imaging Graph 34:69-78, 2010

52. Sapareto SA, Dewey WC: Thermal dose determination in cancer therapy. Int J Radiat Oncol Biol Phys 10:787-800, 1984

53. Schueller G, Kettenbach J, Sedivy R, Bergmeister H, Stift A, Fried J, et al: Expression of heat shock proteins in human hepatocellular carcinoma after radiofrequency ablation in an animal model. Oncol Rep 12:495-499, 2004

54. Shimizu J, Fukuda T, Abe T, Ogihara M, Kubota J, Sasaki A, et al: Ultrasound safety with midfrequency transcranial sonothrombolysis: preliminary study on normal macaca monkey brain. Ultrasound Med Biol 38:1040-1050, 2012

55. Sokka SD, King R, Hynynen K: MRI-guided gas bubble enhanced ultrasound heating in in vivo rabbit thigh. Phys Med Biol 48:223-241, 2003

56. Tanter M, Pernot M, Aubry JF, Montaldo G, Marquet F, Fink $\mathrm{M}$ : Compensating for bone interfaces and respiratory motion in high-intensity focused ultrasound. Int J Hyperthermia 23:141-151, 2007

57. Tung YS, Vlachos F, Choi JJ, Deffieux T, Selert K, Konofagou EE: In vivo transcranial cavitation threshold detection during ultrasound-induced blood-brain barrier opening in mice. Phys Med Biol 55:6141-6155, 2010

58. Wu F, Wang ZB, Lu P, Xu ZL, Chen WZ, Zhu H, et al: Activated anti-tumor immunity in cancer patients after high intensity focused ultrasound ablation. Ultrasound Med Biol 30:1217-1222, 2004

59. Wu J, Ross JP, Chiu JF: Reparable sonoporation generated by microstreaming. J Acoust Soc Am 111:1460-1464, 2002

60. Yin X, Hynynen K: A numerical study of transcranial focused ultrasound beam propagation at low frequency. Phys Med Biol 50:1821-1836, 2005

61. Younan Y, Deffieux T, Larrat B, Fink M, Tanter M, Aubry JF: Influence of the pressure field distribution in transcranial ultrasonic neurostimulation. Med Phys 40:082902, 2013

\section{Author Contributions}

Conception and design: Sheehan, Xu, Aubry. Acquisition of data: Xu, Carlson, Snell, Lopes, Raghavan, Aubry. Analysis and interpretation of data: Sheehan, Xu, Carlson, Eames, Lopes, Aubry. Drafting the article: Sheehan, Xu, Carlson, Snell, Eames, Hananel, Lopes, Raghavan, Lee, Yen, Schlesinger, Aubry.

Critically revising the article: Sheehan, Xu, Carlson, Snell, Lopes, Aubry. Reviewed submitted version of manuscript: Sheehan, Xu, Snell, Eames, Hananel, Lopes, Raghavan, Lee, Yen, Schlesinger, Kassell, Aubry. Approved the final version of the manuscript on behalf of all authors: Sheehan. Statistical analysis: Xu, Carlson. Administrative/technical/material support: Sheehan, Xu. Study supervision: Sheehan, Xu.

\section{Correspondence}

Jason Sheehan, Department of Neurological Surgery, University of Virginia, Box 800212, Charlottesville, VA 22908. email: jsheehan@virginia.edu. 